\title{
Message from the Guest Editor of the 17th Multiphase Flow Conference Special Issue
}

\author{
Dirk Lucas (ه) \\ Institute of Fluid Dynamics, Helmholtz-Zentrum Dresden-Rossendorf, Bautzner Landstr. 400, 01328 Dresden, Germany \\ ๑) Tsinghua University Press 2020
}

Dear readers,

I am very pleased that the Experimental and Computational Multiphase Flow (ECMF) journal again gives us the opportunity to publish selected papers from our annual Multiphase Flow Workshop. While several papers based on contributions to the 17th Multiphase Flow Conference are published in regular issues of the Journal, six papers can be found in this Special Issue.

Since 2003, the Multiphase Flow Conference and Short Course takes place annually in Dresden (Germany) and is jointly organized by Helmholtz-Zentrum Dresden-Rossendorf (HZDR) and ANSYS. It aims to bring together experts on numerics and CFD-modelling with experimentalists and specialists on two-phase flow measuring techniques as well as to cover a wide range from the basics of multiphase flows to multifaceted industrial applications. For the 17th edition of this event in November 2019, HZDR could attract ANSYS, Siemens PLM, the OpenFOAM Foundation, ISimQ GmbH, and the Virtual International Research Institute of Two-Phase Flow and Heat Transfer as partners. Two-day short course was followed by a two-day conference. 78 contributions were presented at the conference part, 2 of them as invited keynote lectures, 27 as oral presentations, and 49 as posters. More abstracts were submitted than could be considered in the programme. This allowed a selection and ensured a very good quality of the contributions. Selected contributions were invited to submit papers to ECMF and six of them are published after a rigorous review process in this Special Issue. They focus on modelling approaches, methodologies, and numerical issues for different multiphase flow problems.

Beside advanced experiments, Direct Numerical Simulations (DNS) become more and more important to gain detailed knowledge on local phenomena determining the interaction between the phases and to develop closure models for EulerLagrange and Euler-Euler approaches. In the paper "Complex bubble deformation and break-up dynamics studies using interface capturing approach" (https://doi.org/10.1007/ s42757-020-0073-3), DNS on single bubbles are presented to study bubble deformation over a wide range of parameters. This includes the peripheral shear off of satellite bubbles from skirt bubbles. A special Proportional-IntegralDerivative (PID) bubble controller is used to keep the bubble at a fixed position.

The paper "Efficient simulation of bubble dispersion and resulting interaction" (https://doi.org/10.1007/s42757020-0082-2), aims to establish a consistent and efficient model setup for bubbly flows in the Euler-Lagrange framework. Here still one-way coupling is used to separate effects. The bubble dispersion due to turbulence of the liquid phase is considered by a continuous random walk model. Special methods are developed to simulate the bubble collisions resulting from the dispersion in an efficient way. Different models for the coalescence efficiency are analysed. The validation shows promising results for the simulation of dense bubbly flows.

Two other papers are related to the Euler-Euler approach. One of them, entitled "Multi-fluid modelling of bubbly channel flows with an adaptive multigroup population balance method" (https://doi.org/10.1007/s42757-020-00845), presents the Adaptive Multiple Size-Group (A-MuSiG) in STAR-CCM+ and its application to different bubbly channel flows. Different kernels for bubble coalescence and breakup are investigated and corresponding empirical parameters are calibrated independently using different experimental databases. Finally, the calibrated model is validated by a complex flow in a channel with an obstacle and the coupling between the bubble size distribution and the evolution of the flow is demonstrated. The other paper, "3D simulation of gas-laden liquid flows in centrifugal pumps and the assessment of two-fluid CFD methods" (https://doi.org/ 10.1007/s42757-020-0080-4), presents a more applicationoriented approach of the Euler-Euler modelling. The performance of pumps sensitively depends on the two-phase flow characteristics. The applicability of the two-fluid model

$\triangle$ D. Lucas@hzdr.de 
is assessed on experimental data for horizontal planar diffusor and for a centrifugal pump. There are clear limitations using the presently available models with the mono-disperse assumption and the need for hybrid models combining disperse flow models with VOF-like (Volume of Fluids) methods is pointed out.

Numerical issues related with such VOF models are discussed in both of the remaining papers of this Special Issue. The implementation of high-resolution schemes for the advection of the volume fraction influences the stability and convergence. The paper "A Modified Normalized Weighting Factor method for improving the efficiency of the blended high-resolution advection schemes in the context of multiphase flows" (https:// doi.org/10.1007/s42757020-0074-2) presents a modification of the normalized weighting factor method, which improves convergence and stability compared to other methods and similar accuracy is obtained with less computational time. The influence of a model parameter on spurious currents is analysed in the paper "On sharp surface force model: Effect of sharpening coefficient" (https://doi.org/10.1007/s42757-020-0063-5). An increase of the sharpening coefficient reduces spurious currents, but too large values may also lead to an unphysical behaviour. The authors conclude that an optimized value has to be used depending on the application.

Unfortunately, caused by the COVID-19 pandemic, we have to skip the multiphase flow workshop in 2020, but we hope to be able to continue in 2021. I am looking forward to a fruitful continuation of this workshop!

Sincerely yours,

Dirk Lucas 